\title{
Improvements of the Hermite-Hadamard inequality
}

\section{Zlatko Pavić}

\section{"Correspondence:} Zlatko.Pavic@sfsb.hr Mechanical Engineering Faculty in Slavonski Brod, University of Osijek, Trg Ivane Brlić Mažuranić 2,

Slavonski Brod, 35000, Croatia

\begin{abstract}
The article provides refinements and generalizations of the Hermite-Hadamard inequality for convex functions on the bounded closed interval of real numbers. Improvements are related to the discrete and integral part of the inequality.
\end{abstract}

MSC: 26A51;26D15

Keywords: convex combination; convex function; the Hermite-Hadamard inequality

\section{Introduction}

Let $\mathbb{X}$ be a real linear space. A linear combination $\alpha a+\beta b$ of points $a, b \in \mathbb{X}$ and coefficients $\alpha, \beta \in \mathbb{R}$ is affine if $\alpha+\beta=1$. A set $\mathcal{S} \subseteq \mathbb{X}$ is affine if it contains all binomial affine combinations of its points. A function $h: \mathcal{S} \rightarrow \mathbb{R}$ is affine if the equality

$$
h(\alpha a+\beta b)=\alpha h(a)+\beta h(b)
$$

holds for every binomial affine combination $\alpha a+\beta b$ of the affine set $\mathcal{S}$.

Convex combinations and sets are introduced by restricting to affine combinations with nonnegative coefficients. A function $h: \mathcal{S} \rightarrow \mathbb{R}$ is convex if the inequality

$$
f(\alpha a+\beta b) \leq \alpha f(a)+\beta f(b)
$$

holds for every binomial convex combination $\alpha a+\beta b$ of the convex set $\mathcal{S}$.

The above concept applies to all $n$-membered affine or convex combinations. Jensen (see [1]) extended the inequality in equation (2) by relying on induction.

\section{Focusing on the set center and barycenter}

We use the real line $\mathbb{X}=\mathbb{R}$. If $\kappa_{1}, \ldots, \kappa_{n} \in \mathbb{R}$ are nonnegative coefficients satisfying $\sum_{i=1}^{n} \kappa_{i}=1$, and if $\mathcal{S}=\left\{x_{1}, \ldots, x_{n}\right\}$ is a set of points $x_{i} \in \mathbb{R}$, then the convex combination point

$$
c=\sum_{i=1}^{n} \kappa_{i} x_{i}
$$

is called the center of the set $\mathcal{S}$ respecting coefficients $\kappa_{i}$, or just the set center.

(c) 2015 Pavić. This article is distributed under the terms of the Creative Commons Attribution 4.0 International License (http://creativecommons.org/licenses/by/4.0/), which permits unrestricted use, distribution, and reproduction in any medium, provided you give appropriate credit to the original author(s) and the source, provide a link to the Creative Commons license, and indicate if changes were made. 
An integral version utilizes a measure. If $\mu$ is a measure on $\mathbb{R}$, and if $\mathcal{S} \subseteq \mathbb{R}$ is a measurable set of positive measure, then the integral mean point

$$
c=\frac{1}{\mu(\mathcal{S})} \int_{\mathcal{S}} x d \mu
$$

is called the barycenter of the set $\mathcal{S}$ respecting measure $\mu$, or just the set barycenter.

In both discrete and integral cases, the point $c$ belongs to the convex hull of the set $\mathcal{S}$, as the smallest convex set containing $\mathcal{S}$.

Throughout the paper we will use a bounded interval of real numbers with endpoints $a<b$. Each point $x \in[a, b]$ can be presented by the unique binomial convex combination

$$
x=\frac{b-x}{b-a} a+\frac{x-a}{b-a} b .
$$

The next three lemmas present the properties of a convex function $f:[a, b] \rightarrow \mathbb{R}$ concerning its supporting and secant lines.

The discrete version refers to interval points and interval endpoints sharing the common center.

Lemma A Let $[a, b] \subset \mathbb{R}$ be a closed interval, and let $\sum_{i=1}^{n} \kappa_{i} x_{i}$ be a convex combination of points $x_{i} \in[a, b]$. Let $\alpha a+\beta b$ be the unique endpoints convex combination such that

$$
\sum_{i=1}^{n} \kappa_{i} x_{i}=\alpha a+\beta b .
$$

Then every convex function $f:[a, b] \rightarrow \mathbb{R}$ satisfies the double inequality

$$
f(\alpha a+\beta b) \leq \sum_{i=1}^{n} \kappa_{i} f\left(x_{i}\right) \leq \alpha f(a)+\beta f(b)
$$

Proof Taking $c=\sum_{i=1}^{n} \kappa_{i} x_{i}$, we have the following two cases.

If $c \in\{a, b\}$, then equation (7) is reduced to $f(c) \leq f(c) \leq f(c)$.

If $c \in(a, b)$, then using a supporting line $y=h_{1}(x)$ of the convex curve $y=f(x)$ at the graph point $C(c, f(c))$, and the secant line $y=h_{2}(x)$ passing through the graph points $A(a, f(a))$ and $B(b, f(b))$, we get the inequality

$$
\begin{aligned}
f(\alpha a+\beta b) & =h_{1}(\alpha a+\beta b)=\sum_{i=1}^{n} \kappa_{i} h_{1}\left(x_{i}\right) \\
& \leq \sum_{i=1}^{n} \kappa_{i} f\left(x_{i}\right) \\
& \leq \sum_{i=1}^{n} \kappa_{i} h_{2}\left(x_{i}\right)=h_{2}(\alpha a+\beta b)=\alpha f(a)+\beta f(b)
\end{aligned}
$$

containing equation (7).

The discrete-integral version refers to the connection of the interval barycenter with interval endpoints. 
Lemma B Let $[a, b] \subset \mathbb{R}$ be a closed interval, and let $\mu$ be a positive measure on $\mathbb{R}$ such that $\mu([a, b])>0$. Let $\alpha a+\beta b$ be the unique endpoints convex combination such that

$$
\frac{1}{\mu([a, b])} \int_{[a, b]} x d \mu=\alpha a+\beta b
$$

Then every convex function $f:[a, b] \rightarrow \mathbb{R}$ satisfies the double inequality

$$
f(\alpha a+\beta b) \leq \frac{1}{\mu([a, b])} \int_{[a, b]} f(x) d \mu \leq \alpha f(a)+\beta f(b) .
$$

Proof The proof coincides with the proof of Lemma A, provided that we use the integral means instead of $n$-membered convex combinations.

The integral version refers to the given interval and its subinterval sharing the common barycenter.

Lemma $\mathbf{C}$ Let $\mathcal{A}, \mathcal{B} \subset \mathbb{R}$ be bounded closed intervals such that $\mathcal{A} \subset \mathcal{B}$. Let $\mu$ be a positive measure on $\mathbb{R}$ such that $0<\mu(\mathcal{A})<\mu(\mathcal{B})$ and

$$
\frac{1}{\mu(\mathcal{A})} \int_{\mathcal{A}} x d \mu=\frac{1}{\mu(\mathcal{B})} \int_{\mathcal{B}} x d \mu
$$

Then every convex function $f: \mathcal{B} \rightarrow \mathbb{R}$ satisfies the double inequality

$$
\frac{1}{\mu(\mathcal{A})} \int_{\mathcal{A}} f(x) d \mu \leq \frac{1}{\mu(\mathcal{B})} \int_{\mathcal{B}} f(x) d \mu \leq \frac{1}{\mu(\mathcal{B} \backslash \mathcal{A})} \int_{\mathcal{B} \backslash \mathcal{A}} f(x) d \mu .
$$

Proof Equation (11) can be extended with the barycenter of the set $\mathcal{B} \backslash \mathcal{A}$. Using the secant line $y=h(x)$ of the convex curve $y=f(x)$ respecting the interval $\mathcal{A}(f(x) \leq h(x), x \in \mathcal{A}$ and $f(x) \geq h(x), x \in \mathcal{B} \backslash \mathcal{A})$, we firstly prove the inequality of the left and right terms of equation (12). Then we express the middle term of equation (12) as the convex combination of the left and right terms.

A functional approach related to the above lemmas can be found in [2]. A more general version of Lemma $\mathrm{C}$ can be found in [3].

Using the Riemann integral in Lemma B, the condition in (9) gives the midpoint

$$
\frac{1}{b-a} \int_{a}^{b} x d x=\frac{1}{2} a+\frac{1}{2} b
$$

and its use in equation (10) implies the classic Hermite-Hadamard inequality

$$
f\left(\frac{a+b}{2}\right) \leq \frac{1}{b-a} \int_{a}^{b} f(x) d x \leq \frac{f(a)+f(b)}{2}
$$

In fact, the above inequality holds for every integrable function $f:[a, b] \rightarrow \mathbb{R}$ that admits a supporting line at the midpoint $c=(a+b) / 2$, and fits into the supporting-secant line inequality

$$
h_{1}(x) \leq f(x) \leq h_{2}(x), \quad x \in[a, b] .
$$


For example, the function $f(x)=\sqrt{|x|}$ is integrable on $[-1,1]$, admits a supporting line at zero, and satisfies equation (15), so it satisfies equation (14). This function is not convex on any subinterval of $[-1,1]$.

Moreover, the inequality in equation (14) follows by integrating the inequality in equation (15) over the interval $[a, b]$.

We finish the section with a historic note on the important Hermite-Hadamard inequality. In 1883, studying convex functions, Hermite (see [4]) attained the inequality in equation (14). In 1893, not knowing Hermite's result, Hadamard (see [5]) got the left-hand side of equation (14). For information as regards this inequality, one may refer to books [6] and [7], and papers [8-11] and [12].

\section{Main results}

To refine the Hermite-Hadamard inequality in equation (14), we will use convex combinations of points of the closed interval $[a, b]$. In the next theorem, we will refine the double inequality in equation (14) by using two convex combinations of the midpoint $x=(a+b) / 2$.

Theorem 3.1 Let $[a, b] \subset \mathbb{R}$ be a closed interval, let $c, d \in[a, b]$ be interval points, and let

$$
\alpha=\frac{c-a}{b-a}, \quad \beta=\frac{b-c}{b-a}, \quad \gamma=\frac{d-a}{b-a}, \quad \delta=\frac{b-d}{b-a} .
$$

Then every convex function $f:[a, b] \rightarrow \mathbb{R}$ satisfies the series of inequalities

$$
\begin{aligned}
f\left(\frac{a+b}{2}\right) & \leq \alpha f\left(\frac{a+c}{2}\right)+\beta f\left(\frac{c+b}{2}\right) \\
& \leq \frac{1}{b-a} \int_{a}^{b} f(x) d x \\
& \leq \frac{\gamma f(a)+\delta f(b)+f(d)}{2} \leq \frac{f(a)+f(b)}{2} .
\end{aligned}
$$

Proof If $c, d \in\{a, b\}$, then the inequality in equation (16) is actually reduced to the Hermite-Hadamard inequality in equation (14).

Suppose that $c \notin\{a, b\}$. Applying equation (5) to the inclusion $(a+b) / 2 \in[(a+c) / 2,(c+$ $b$ )/2], we get the convex combination equality

$$
\frac{a+b}{2}=\alpha \frac{a+c}{2}+\beta \frac{c+b}{2} .
$$

Applying the convexity of $f$ to the right-hand side of equation (17), and the left-hand side of the Hermite-Hadamard inequality to midpoints $(a+c) / 2$ and $(c+b) / 2$, we get

$$
\begin{aligned}
f\left(\frac{a+b}{2}\right) & \leq \alpha f\left(\frac{a+c}{2}\right)+\beta f\left(\frac{c+b}{2}\right) \\
& \leq \frac{1}{b-a} \int_{a}^{c} f(x) d x+\frac{1}{b-a} \int_{c}^{b} f(x) d x \\
& =\frac{1}{b-a} \int_{a}^{b} f(x) d x
\end{aligned}
$$

proving the first half of equation (16). 
Suppose that $d \notin\{a, b\}$. In this case, we will use the convex combination equality

$$
\frac{\gamma}{2} a+\frac{\delta}{2} b+\frac{1}{2} d=\frac{1}{2} a+\frac{1}{2} b
$$

in terms of equation (6). Applying the right-hand side of the Hermite-Hadamard inequality to intervals $[a, d]$ and $[d, b]$, and Lemma A to the combination in (19), we obtain

$$
\begin{aligned}
\frac{1}{b-a} \int_{a}^{b} f(x) d x & =\frac{1}{b-a} \int_{a}^{d} f(x) d x+\frac{1}{b-a} \int_{d}^{b} f(x) d x \\
& \leq \frac{\gamma}{2} f(a)+\frac{\delta}{2} f(b)+\frac{1}{2} f(d) \\
& \leq \frac{1}{2} f(a)+\frac{1}{2} f(b)
\end{aligned}
$$

proving the second half of equation (16).

If $c=d=(a+b) / 2$, equation (16) takes the form

$$
\begin{aligned}
f\left(\frac{a+b}{2}\right) & \leq \frac{1}{2}\left[f\left(\frac{3 a+b}{4}\right)+f\left(\frac{a+3 b}{4}\right)\right] \\
& \leq \frac{1}{b-a} \int_{a}^{b} f(x) d x \\
& \leq \frac{1}{2}\left[f\left(\frac{a+b}{2}\right)+\frac{f(a)+f(b)}{2}\right] \leq \frac{f(a)+f(b)}{2}
\end{aligned}
$$

The above improvement of the Hermite-Hadamard inequality was specified in [7].

Now we estimate the double inequality of equation (16) containing the integral term. Taking $d=c$ and using the arithmetic mean form, we can find that the following estimation holds.

Corollary 3.2 Let $[a, b] \subset \mathbb{R}$ be a closed interval, let $c \in[a, b]$ be an interval point, and let

$$
\alpha=\frac{c-a}{b-a}, \quad \beta=\frac{b-c}{b-a} .
$$

Then every convex function $f:[a, b] \rightarrow \mathbb{R}$ satisfies the inequality

$$
\frac{1}{b-a} \int_{a}^{b} f(x) d x \leq \frac{1}{2}\left(\alpha f\left(\frac{a+c}{2}\right)+\beta f\left(\frac{c+b}{2}\right)\right)+\frac{1}{2} \frac{\alpha f(a)+\beta f(b)+f(c)}{2} .
$$

Proof If $c \in\{a, b\}$, the inequality in equation (22) yields the well-known estimation

$$
\frac{1}{b-a} \int_{a}^{b} f(x) d x \leq \frac{1}{2} f\left(\frac{a+b}{2}\right)+\frac{1}{2} \frac{f(a)+f(b)}{2}
$$

We now suppose that $c \in(a, b)$. From the series of inequalities in equation (16), we extract the second last inequality referring to the inclusion $d \in[a, b]$ as

$$
\frac{2}{b-a} \int_{a}^{b} f(x) d x \leq \frac{d-a}{b-a} f(a)+\frac{b-d}{b-a} f(b)+f(d) .
$$


Applying the above inequality to inclusions $(a+c) / 2 \in[a, c]$ and $(c+b) / 2 \in[c, b]$, we get

$$
\begin{aligned}
\frac{2}{b-a} \int_{a}^{b} f(x) d x & =\frac{c-a}{b-a}\left[\frac{2}{c-a} \int_{a}^{c} f(x) d x\right]+\frac{b-c}{b-a}\left[\frac{2}{b-c} \int_{c}^{b} f(x) d x\right] \\
& \leq \alpha\left[\frac{1}{2} f(a)+\frac{1}{2} f(c)+f\left(\frac{a+c}{2}\right)\right]+\beta\left[\frac{1}{2} f(c)+\frac{1}{2} f(b)+f\left(\frac{c+b}{2}\right)\right] \\
& =\alpha f\left(\frac{a+c}{2}\right)+\beta f\left(\frac{c+b}{2}\right)+\frac{\alpha f(a)+\beta f(b)+f(c)}{2},
\end{aligned}
$$

and dividing by 2 , we obtain the inequality in equation (22).

The interpolated terms in equation (16) can be expanded.

Corollary 3.3 Let $[a, b] \subset \mathbb{R}$ be a closed interval, let $c_{1}, \ldots, c_{n} \in[a, b]$ and $d_{1}, \ldots, d_{m} \in[a, b]$ be interval points, and let

$$
\alpha_{i}=\frac{c_{i}-a}{n(b-a)}, \quad \beta_{i}=\frac{b-c_{i}}{n(b-a)}, \quad \gamma_{j}=\frac{d_{j}-a}{m(b-a)}, \quad \delta_{j}=\frac{b-d_{j}}{m(b-a)} .
$$

Then every convex function $f:[a, b] \rightarrow \mathbb{R}$ satisfies the series of inequalities

$$
\begin{aligned}
f\left(\frac{a+b}{2}\right) & \leq \sum_{i=1}^{n}\left[\alpha_{i} f\left(\frac{a+c_{i}}{2}\right)+\beta_{i} f\left(\frac{c_{i}+b}{2}\right)\right] \\
& \leq \frac{1}{b-a} \int_{a}^{b} f(x) d x \\
& \leq \frac{\sum_{j=1}^{m}\left[\gamma_{j} f(a)+\delta_{j} f(b)+m^{-1} f\left(d_{j}\right)\right]}{2} \leq \frac{f(a)+f(b)}{2}
\end{aligned}
$$

Proof The inequality in equation (26) can be achieved by including the convex combinations

$$
\frac{a+b}{2}=\sum_{i=1}^{n}\left[\alpha_{i} \frac{a+c_{i}}{2}+\beta_{i} \frac{c_{i}+b}{2}\right]
$$

and

$$
\sum_{j=1}^{m}\left[\frac{\gamma_{j}}{2} a+\frac{\delta_{j}}{2} b+\frac{1}{2 m} d_{j}\right]=\frac{1}{2} a+\frac{1}{2} b
$$

to the procedure of the proof of Theorem 3.1.

The following theorem presents a generalization of the Hermite-Hadamard inequality to any point of the open interval $(a, b)$.

Theorem 3.4 Let $[a, b] \subset \mathbb{R}$ be a closed interval, and let $\alpha a+\beta b$ be the endpoints convex combination whose coefficients are positive. 
Then every convex function $f:[a, b] \rightarrow \mathbb{R}$ satisfies the series of inequalities

$$
\begin{aligned}
f(\alpha a+\beta b) & \leq \alpha f\left(\frac{a+\alpha a+\beta b}{2}\right)+\beta f\left(\frac{\alpha a+\beta b+b}{2}\right) \\
& \leq \frac{\alpha}{\beta(b-a)} \int_{a}^{\alpha a+\beta b} f(x) d x+\frac{\beta}{\alpha(b-a)} \int_{\alpha a+\beta b}^{b} f(x) d x \\
& \leq \frac{f(\alpha a+\beta b)}{2}+\frac{\alpha f(a)+\beta f(b)}{2} \leq \alpha f(a)+\beta f(b) .
\end{aligned}
$$

Proof Applying equation (5) to the inclusion $\alpha a+\beta b \in[(a+\alpha a+\beta b) / 2,(\alpha a+\beta b+b) / 2]$, we get the convex combination equality

$$
\alpha a+\beta b=\alpha \frac{a+\alpha a+\beta b}{2}+\beta \frac{\alpha a+\beta b+b}{2} .
$$

Applying the convexity of $f$ to the right-hand side of equation (30), and the left-hand side of the Hermite-Hadamard inequality to midpoints $(a+\alpha a+\beta b) / 2$ and $(\alpha a+\beta b+b) / 2$, we get the first half of equation (29).

Applying the right-hand side of the Hermite-Hadamard inequality to intervals $[a, \alpha a+$ $\beta b]$ and $[\alpha a+\beta b, b]$, and the convexity inequality in equation (2), we obtain the second half of equation (29).

If $\alpha=\beta=1 / 2$, then the inequality in equation (29) is reduced to the inequality in equation (21).

The integral refinements of the Hermite-Hadamard inequality can be obtained by applying Lemma C.

Theorem 3.5 Let $[a, b] \subset \mathbb{R}$ be a closed interval, and let $\delta$ be a positive number less than $(b-a) / 2$.

Then every convex function $f:[a, b] \rightarrow \mathbb{R}$ satisfies the series of inequalities

$$
\begin{aligned}
f\left(\frac{a+b}{2}\right) & \leq \frac{1}{b-a-2 \delta} \int_{a+\delta}^{b-\delta} f(x) d x \\
& \leq \frac{1}{b-a} \int_{a}^{b} f(x) d x \\
& \leq \frac{1}{2 \delta}\left(\int_{a}^{a+\delta} f(x) d x+\int_{b-\delta}^{b} f(x) d x\right) \leq \frac{f(a)+f(b)}{2}
\end{aligned}
$$

Proof Let $\mathcal{A}=[a+\delta, b-\delta]$ and $\mathcal{B}=[a, b]$ be observed intervals, and let $|\mathcal{A}|$ and $|\mathcal{B}|$ be their lengths, respectively. The barycenter of the sets $\mathcal{A}, \mathcal{B}$ and $\mathcal{B} \backslash \mathcal{A}$ falls into the midpoint $c=(a+b) / 2$. Let $y=h_{1}(x)$ be the supporting line of the curve $y=f(x)$ at the graph point $C(c, f(c))$, and let $y=h_{2}(x)$ be the secant line passing through the graph points $A(a+\delta, f(a+$ $\delta))$ and $B(b-\delta, f(b-\delta))$.

To prove the first inequality of equation (31), we use the supporting line

$$
f\left(\frac{a+b}{2}\right)=h_{1}\left(\frac{a+b}{2}\right)=\frac{1}{|\mathcal{A}|} \int_{\mathcal{A}} h_{1}(x) d x \leq \frac{1}{|\mathcal{A}|} \int_{\mathcal{A}} f(x) d x
$$


To prove the last inequality of equation (31), we use the secant line

$$
\frac{1}{|\mathcal{B} \backslash \mathcal{A}|} \int_{\mathcal{B} \backslash \mathcal{A}} f(x) d x \leq \frac{1}{|\mathcal{B} \backslash \mathcal{A}|} \int_{\mathcal{B} \backslash \mathcal{A}} h_{2}(x) d x=h_{2}\left(\frac{a+b}{2}\right)=\frac{f(a)+f(b)}{2} .
$$

The double inequality of equation (31) containing the integrals follows from the inequality in equation (12).

Considerations similar to those in Corollary 3.2 can be carried out for equations (26), (29) and (31).

\section{Refinements of the most important means}

Thorough this section we use positive numbers $a$ and $b$, positive coefficients $\alpha$ and $\beta$ whose sum is equal to 1 , and a strictly monotone continuous function $\varphi:[a, b] \rightarrow \mathbb{R}$.

The discrete quasi-arithmetic mean of the numbers $a$ and $b$ with the coefficients $\alpha$ and $\beta$ respecting the function $\varphi$ is defined by the number

$$
M_{\varphi}(a, b ; \alpha, \beta)=\varphi^{-1}(\alpha \varphi(a)+\beta \varphi(b)) .
$$

Using the identity function $\varphi(x)=x$, we get the generalized arithmetic mean

$$
A(a, b ; \alpha, \beta)=\alpha a+\beta b,
$$

using the hyperbolic function $\varphi(x)=1 / x$, we have the generalized harmonic mean

$$
H(a, b ; \alpha, \beta)=\left(\alpha a^{-1}+\beta b^{-1}\right)^{-1},
$$

and using the logarithmic function $\varphi(x)=\ln x$, we obtain the generalized geometric mean

$$
G(a, b ; \alpha, \beta)=a^{\alpha} b^{\beta} .
$$

The above means satisfy the generalized harmonic-geometric-arithmetic mean inequality

$$
H(a, b ; \alpha, \beta)<G(a, b ; \alpha, \beta)<A(a, b ; \alpha, \beta) .
$$

Applying equation (29) to the convex function $f(x)=-\ln x$ using substitutions $a \mapsto 1 / a$ and $b \mapsto 1 / b$, and then acting on the rearranged inequality with the exponential function, we can derive the series of inequalities

$$
\begin{aligned}
H(a, b ; \alpha, \beta) & \leq\left[H\left(a, b ; \frac{\alpha+1}{2}, \frac{\beta}{2}\right)\right]^{\alpha}\left[H\left(a, b ; \frac{\alpha}{2}, \frac{\beta+1}{2}\right)\right]^{\beta} \\
& \leq e e^{\frac{\alpha}{\beta} \frac{b}{b-a}} b^{\frac{\beta}{\alpha} \frac{a}{a-b}}\left(\alpha a^{-1}+\beta b^{-1}\right)^{\frac{\alpha-\beta}{\alpha \beta} \frac{\alpha b+\beta a}{b-a}} \\
& \leq[H(a, b ; \alpha, \beta) G(a, b ; \alpha, \beta)]^{\frac{1}{2}} \leq G(a, b ; \alpha, \beta)
\end{aligned}
$$

refining the generalized harmonic-geometric mean inequality. 
Applying equation (29) to the exponential function $f(x)=e^{x}$ using substitutions $a \mapsto \ln a$ and $b \mapsto \ln b$, we can obtain the series of inequalities

$$
\begin{aligned}
G(a, b ; \alpha, \beta) & \leq \alpha G\left(a, b ; \frac{\alpha+1}{2}, \frac{\beta}{2}\right)+\beta G\left(a, b ; \frac{\alpha}{2}, \frac{\beta+1}{2}\right) \\
& \leq \frac{\frac{\alpha}{\beta} a-\frac{\beta}{\alpha} b-\frac{\alpha-\beta}{\alpha \beta} a^{\alpha} b^{\beta}}{\ln a-\ln b} \\
& \leq \frac{G(a, b ; \alpha, \beta)+A(a, b ; \alpha, \beta)}{2} \leq A(a, b ; \alpha, \beta)
\end{aligned}
$$

refining the generalized geometric-arithmetic mean inequality.

To denote the elementary means with coefficients $\alpha=\beta=1 / 2$, we will use the abbreviations $A(a, b), H(a, b)$ and $G(a, b)$.

The integral quasi-arithmetic mean of the numbers $a$ and $b$ respecting the function $\varphi$ is the number

$$
M_{\varphi}(a, b)=\varphi^{-1}\left(\frac{1}{b-a} \int_{a}^{b} \varphi(x) d x\right)
$$

Using the identity function, we get the arithmetic mean

$$
A(a, b)=\frac{1}{b-a} \int_{a}^{b} x d x=\frac{a+b}{2}
$$

using the hyperbolic function, we have the logarithmic mean

$$
L(a, b)=\left(\frac{1}{b-a} \int_{a}^{b} \frac{1}{x} d x\right)^{-1}=\frac{b-a}{\ln b-\ln a},
$$

and using the logarithmic function, we obtain the identric mean

$$
I(a, b)=\exp \left(\frac{1}{b-a} \int_{a}^{b} \ln x d x\right)=\frac{1}{e}\left(\frac{b^{b}}{a^{a}}\right)^{\frac{1}{b-a}} .
$$

The well-known mean inequality says that

$$
H(a, b)<G(a, b)<L(a, b)<I(a, b)<A(a, b) .
$$

Applying equation (16) to the function $f(x)=-\ln x$, using substitutions $a \mapsto 1 / a, b \mapsto$ $1 / b, c \mapsto 1 / c$ and $d \mapsto 1 / d$, and then acting on the rearranged inequality with the exponential function, we can obtain the refined harmonic-geometric mean inequality

$$
\begin{aligned}
H(a, b) & \leq 2 c\left(\frac{a}{a+c}\right)^{\frac{b(c-a)}{c(b-a)}}\left(\frac{b}{c+b}\right)^{\frac{a(b-c)}{c(b-a)}} \\
& \leq\left[I\left(a^{-1}, b^{-1}\right)\right]^{-1} \\
& \leq a^{\frac{b(d-a)}{2 d(b-a)}} b^{\frac{a(b-d)}{2 d(b-a)}} d^{\frac{1}{2}} \leq G(a, b) .
\end{aligned}
$$


Applying the first half of the inequality in equation (16) to the exponential function $f(x)=e^{x}$, using substitutions $a \mapsto \ln a, b \mapsto \ln b$ and $c \mapsto \ln c$, we get the refined geometriclogarithmic mean inequality

$$
G(a, b) \leq \frac{\ln c-\ln a}{\ln b-\ln a} \sqrt{a c}+\frac{\ln b-\ln c}{\ln b-\ln a} \sqrt{c b} \leq L(a, b) .
$$

To prove the logarithmic-identric mean inequality $L(a, b)<I(a, b)$, we can apply the integral form of Jensen's inequality (see [13])

$$
f\left(\frac{1}{b-a} \int_{a}^{b} g(x) d x\right) \leq \frac{1}{b-a} \int_{a}^{b} f(g(x)) d x
$$

to the functions $f(x)=-\ln x$ and $g(x)=1 / x$, and then act on the rearranged inequality with the exponential function. To refine the logarithmic-identric mean inequality, we can use the procedure applied in [14].

Applying the first half of the reverse inequality in equation (16) to the concave function $f(x)=\ln x$, and then acting on the rearranged inequality with the exponential function, we have the refined identric-arithmetic mean inequality

$$
I(a, b) \leq \frac{1}{2}(a+c)^{\frac{c-a}{b-a}}(c+b)^{\frac{b-c}{b-a}} \leq A(a, b) .
$$

\section{Quasi-arithmetic version of the Hermite-Hadamard inequality}

The following is the generalization of Lemma $B$ that includes a strictly monotone continuous function. In this generalization, we use the Riemann integral.

Lemma 5.1 Let $[a, b] \subset \mathbb{R}$ be a closed interval, and let $\varphi:[a, b] \rightarrow \mathbb{R}$ be a strictly monotone continuous function. Let $\alpha \varphi(a)+\beta \varphi(b)$ be the unique convex combination of endpoints of the interval $\varphi([a, b])$ such that

$$
\frac{1}{b-a} \int_{a}^{b} \varphi(x) d x=\alpha \varphi(a)+\beta \varphi(b)
$$

Then every convex function $f$ whose domain contains the image of $\varphi$ satisfies the double inequality

$$
f(\alpha \varphi(a)+\beta \varphi(b)) \leq \frac{1}{b-a} \int_{a}^{b} f(\varphi(x)) d x \leq \alpha f(\varphi(a))+\beta f(\varphi(b)) .
$$

Proof We put $a_{1}=\varphi(a), b_{1}=\varphi(b)$, and $c_{1}=\alpha a_{1}+\beta b_{1}$. The point $c_{1}$ belongs to the interior of the interval $\varphi([a, b])$. Let $z=h_{1}(y)$ be a supporting line of the curve $z=f(y)$ at the graph point $C\left(c_{1}, f\left(c_{1}\right)\right)$, and let $z=h_{2}(y)$ be the secant line passing through the graph points $A\left(a_{1}, f\left(a_{1}\right)\right)$ and $B\left(b_{1}, f\left(b_{1}\right)\right)$. Applying the procedure of proving equation (8) with the use of equalities

$$
h_{1,2}\left(\frac{1}{b-a} \int_{a}^{b} \varphi(x) d x\right)=\frac{1}{b-a} \int_{a}^{b} h_{1,2}(\varphi(x)) d x,
$$

we obtain the double inequality in equation (51). 
To present the quasi-arithmetic version of the Hermite-Hadamard inequality, we need one more notation. If $\varphi$ and $\psi$ are strictly monotone continuous functions on the common interval, then it is said that $\psi$ is $\varphi$-convex if the composition function $\psi \circ \varphi^{-1}$ is convex. The same notation is used for concavity.

Theorem 5.2 Let $[a, b] \subset \mathbb{R}$ be a closed interval, and let $\varphi, \psi:[a, b] \rightarrow \mathbb{R}$ be strictly monotone continuous functions. Let $\alpha \varphi(a)+\beta \varphi(b)$ be the unique binomial convex combination such that

$$
M_{\varphi}(a, b)=M_{\varphi}(a, b ; \alpha, \beta) .
$$

If $\psi$ is either $\varphi$-convex and increasing or $\varphi$-concave and decreasing, then

$$
M_{\varphi}(a, b ; \alpha, \beta) \leq M_{\psi}(a, b) \leq M_{\psi}(a, b ; \alpha, \beta) .
$$

If $\psi$ is either $\varphi$-convex and decreasing or $\varphi$-concave and increasing, then the reverse inequality is valid in equation (54).

Proof We prove the case that $\psi$ is $\varphi$-convex and increasing. The condition in equation (53) actually represents the equality in equation (50) which enables us to use the inequality in equation (51) with the convex function $f=\psi \circ \varphi^{-1}$, and get

$$
\left(\psi \circ \varphi^{-1}\right)(\alpha \varphi(a)+\beta \varphi(b)) \leq \frac{1}{b-a} \int_{a}^{b} \psi(x) d x \leq \alpha \psi(a)+\beta \psi(b)
$$

Acting on the above inequality with the increasing function $\psi$, we obtain the quasiarithmetic mean inequality in equation (54).

\section{Competing interests}

The author declares that they have no competing interests.

\section{Acknowledgements}

This work has been fully supported by Mechanical Engineering Faculty in Slavonski Brod, and Croatian Science Foundation under the project 5435.

Received: 22 January 2015 Accepted: 24 June 2015 Published online: 11 July 2015

\section{References}

1. Jensen, JLWV: Om konvekse Funktioner og Uligheder mellem Middelværdier. Nyt Tidsskr. Math. 16, 49-68 (1905)

2. Pavić, Z: Functions like convex functions. J. Funct. Spaces 2014, Article ID 919470 (2014)

3. Pavić, Z, Pečarić, J, Perić, I: Integral, discrete and functional variants of Jensen's inequality. J. Math. Inequal. 5, 253-264 (2011)

4. Hermite, C: Sur deux limites d'une intégrale définie. Mathesis 3, 82 (1883)

5. Hadamard, J: Étude sur les propriétés des fonctions entières et en particulier d'une fonction considerée par Riemann. J. Math. Pures Appl. 58, 171-215 (1893)

6. Dragomir, SS, Pearce, CEM: Selected Topics on Hermite-Hadamard Inequalities and Applications. RGMIA Monographs. Victoria University, Melbourne (2000)

7. Niculescu, CP, Persson, LE: Convex Functions and Their Applications. Springer, New York (2006)

8. Chen, F: A note on Hermite-Hadamard inequalities for products of convex functions. J. Appl. Math. 2013, Article ID 935020 (2013)

9. El Farissi, A: Simple proof and refinement of Hermite-Hadamard inequality. J. Math. Inequal. 4, 365-369 (2010)

10. Lyu, SL: On the Hermite-Hadamard inequality for convex functions of two variables. Numer. Algebra Control Optim. 4, 1-8 (2014)

11. Niculescu, CP, Persson, LE: Old and new on the Hermite-Hadamard inequality. Real Anal. Exch. 29, 663-685 (2003)

12. Wang, J, Li, X, Fečkan, M, Zhou, Y: Hermite-Hadamard-type inequalities for Riemann-Liouville fractional integrals via two kinds of convexity. Appl. Anal. 92, 2241-2253 (2013)

13. Jensen, JLWV: Sur les fonctions convexes et les inégalités entre les valeurs moyennes. Acta Math. 30, 175-193 (1906)

14. Mićić, J, Pavić, Z, Pečarić, J: The inequalities for quasiarithmetic means. Abstr. Appl. Anal. 2012, Article ID 203145 (2012) 